\title{
Monitoramento nutricional do trigo através do índice de balanço nutricional-DRIS
}

\author{
Nutritional monitoring of wheat by using the index of \\ nutritional balance-DRIS
}

\author{
Lucimara Aparecida Martins ${ }^{1}$; Roberto Antunes Fioretto ${ }^{2 *}$; \\ Inês Cristina Batista Fonseca² ${ }^{2}$ Cristine E. Alvarenga Carneiro ${ }^{3}$
}

Resumo

\begin{abstract}
Deficiências nutricionais na cultura do trigo causam sérios danos à produtividade e à qualidade. Freqüentemente a adubação é focada nos macronutrientes, não levando em consideração a necessidade dos micronutrientes no desenvolvimento da planta. Visando avaliar a intervenção nutricional pelo método do DRIS em uma cultura de trigo já instalada, conduziu-se um experimento de campo em Latossolo Vermelho, em Cândido Mota, SP. O delineamento experimental foi inteiramente casualizado, com três tratamentos e quatro repetições. Todos os tratamentos receberam adubação de semeadura de $250 \mathrm{~kg} \mathrm{ha}$ ${ }^{1}$ de 08-20-20, o tratamento Regional recebeu mais $40 \mathrm{~kg} \mathrm{ha}^{-1}$ de nitrogênio após 30 dias após emergência (dae) e o Monitorado, suplementação nutricional de acordo com as análises do DRIS a cada 30 dias. Aplicou-se no tratamento Monitorado cálcio e boro aos 30 dae, nitrogênio e boro aos 60 dae e fósforo e boro aos 90 dae. $\mathrm{O}$ tratamento Monitorado proporcionou aumento de $21,80 \%$ na produção em relação à adubação Regional. A interferência nutricional na cultura através do DRIS proporcionou melhorias nutricionais, bem como aumento de produção.
\end{abstract}

Palavras-chave: Adubação foliar, intervenção nutricional, produtividade, deficiência

\begin{abstract}
Nutricional deficiency in wheat crop causes serious damages to the productivity and the quality. Often the fertilization is focused in the macronutrients, not accounting the necessity of the micronutrients in the development of the plant. In order to evaluate the nutritional intervention using DRIS method in a wheat crop already installed, an experiment was set on a soil classief as "Latossolo Vermelho", in Cândido Mota, SP. The experimental design was totally randomized, with three treatments and four replications. All treatments received fertilization at sowing with $250 \mathrm{~kg} \mathrm{ha}^{-1}$ of $08-20-20$ formulation. The Regional treatment received more $40 \mathrm{~kg} \mathrm{ha}^{-1}$ of nitrogen after 30 days of emergency (dae) and the monitored one, received a nutritional supplement in agreement with DRIS analysis each 30 days after emergency. In the monitored treatment it was applied calcium and boron at 30 dae, nitrogen and boron at 60 dae and phosphorus and boron at 90 dae. The monitored treatment yielded $21.80 \%$ more than the regional one. The nutrional interference through DRIS provided nutritional and yield improvements in wheat crop.
\end{abstract}

Key words: Leaves fertilization. Nutritional intervention. Productivity. Deficiency.

1 Eng. Agrônoma, aluna do Programa de Pós-graduação em Agronomia-UEL, Área de Concentração Solos. Londrina-PR. E-mail: luci-martins@bol.com.br

2 Eng. Agrônomos, Professores Drs. do Departamento de Agronomia, UEL, Londrina-PR. E-mail roberto@laborsolo.com.br; inescbf@uel.br

3 Química, aluna do Programa de Pós-graduação em Agronomia - UEL, Área de Concentração Solos. Londrina-PR. Bolsista - CNPq.

* Autor para correspondência. 


\section{Introdução}

O resgate da cultura do trigo vem ocorrendo no Estado de São Paulo pelo próprio mercado devido à importância econômica e estratégica, representando uma oportunidade de renda ao agricultor no período de inverno e contribuindo para a sustentação do agronegócio (INSTITUTO AGRÔNOMICO DE CAMPINAS, 2002).

Nutricionalmente, as plantas de trigo demandam maior quantidade dos macronutrientes, nitrogênio e potássio (PAULETTI, 1998). O nitrogênio é um elemento essencial, o qual proporciona alto rendimento e qualidade no trigo. Sua absorção é determinada em grande parte pela disponibilidade e demanda durante vários estádios fenológicos (CAMPBELL; DE JONG, 2000). O trigo cultivado no Brasil, quando estabelecido em sucessão a outras gramíneas, tem na deficiência do $\mathrm{N}$ o fator que mais limita o rendimento de grãos, seguida pela deficiência de $\mathrm{P}$ (FREITAS et al., 2000). Uma alternativa para o aumento da produtividade, é a aplicação de nitrogênio em cobertura, pois os genótipos de trigo apresentam variabilidade nas respostas a aplicação do elemento, isto possibilitará o fornecimento do nitrogênio no momento de maior consumo pela planta, alterando o rendimento de grãos ( FREITAS et al., 1995).

É comum na implantação da lavoura a preocupação com a adubação dos macronutrientes primários; nitrogênio, fósforo e potássio, negligenciando por toda safra os demais elementos essenciais. Furlani et al. (2003) detectaram que o teor limite para deficiência de B nas folhas, foi de 25 $\mathrm{mg} \mathrm{kg}{ }^{-1}$ para todas as cultivares testadas e que, exceto para o tratamento com a mais alta concentração de $\mathrm{B}$, os teores e conteúdos de $\mathrm{P}, \mathrm{Ca}$, $\mathrm{K}$ e $\mathrm{Mg}$ encontrados nas plantas estavam dentro da normalidade e não variaram com os tratamentos. Hoje novas tecnologias podem auxiliar na qualidade e produtividade dos grãos. A diagnose foliar pode avaliar o estado e o equilíbrio nutricional de uma planta, uma vez que o teor do nutriente é resultante da ação e da interação dos fatores que influem em sua disponibilidade no solo e sua absorção pela planta (PAULETTI, 1998).
O Sistema Integrado de Diagnose e Recomendação (DRIS), desenvolvido por Beaufils (1973), tem sido apontado como uma alternativa para a interpretação do estado nutricional das plantas (SUMNER apud SILVA; NOGUEIRA; GUIMARÃES, 2003), apresentando como vantagem o fato de minimizar os efeitos de diluição e de concentrações dos nutrientes. Este método avalia mais precisamente as interações nutricionais através do Índice de Balanço Nutricional, informando a ordem de limitação dos nutrientes, tanto por deficiência, quanto por excesso, além da intensidade da exigência.

O objetivo do trabalho foi avaliar a intervenção nutricional pelo método do DRIS, em uma cultura de trigo já instalada.

\section{Material e Métodos}

O trabalho foi locado em uma área de trigo já instalada, dentro do Campo de Difusão de Tecnologia - Coopermota, na cidade de Cândido Mota SP, com altitude de 490 metros, latitude $22^{\circ} 44^{\prime \prime S ~ e ~ l o n g i t u d e ~}$ $50^{\circ} 23^{\prime} \mathrm{W}$. O solo na área foi classificado como Latossolo Vermelho Eutroférrico (EMPRESA BRASILEIRA DE PESQISA AGROPECUÁRIA, 1999), cujas características químicas foram analisadas segundo Pavan et al. (1992), e os resultados encontram-se na Tabela 1 . Os dados pluviométricos são apresentados na Tabela 2 .

A cultivar de trigo utilizado foi IAC 350 , a adubação de semeadura foi de $250 \mathrm{~kg} \mathrm{ha}^{-1}$ de 08-2020 , recomendada para a região (COMISSÕES CENTRO-SUL BRASILEIRA DE PESQUISA DE TRIGO E TRITICALE, 2004). O espaçamento entre linhas foi de $16 \mathrm{~cm}$, com densidade de sementes na linha de 50 plantas $\mathrm{m}^{-1}$, sendo semeado no dia 29 de abril de 2004. As sementes estavam tratadas com inseticida Imidacloprid na dose de $1 \mathrm{~mL} \mathrm{~kg}^{-1}$ de semente, e Tolylfluanid a $1,5 \mathrm{~g} \mathrm{~kg}^{-1}$ de semente; no controle de pragas utilizou-se duas aplicações de Metamidofos a $200 \mathrm{~mL} \mathrm{ha}^{-1}$, para as plantas daninhas Metsulfuron $6 \mathrm{~g} \mathrm{ha}^{-1}$ e para doenças, Azoxystrobim mais Cyproconazole a $300 \mathrm{~mL} \mathrm{ha}^{-1}$. 
Tabela 1. Características químicas do solo do experimento.

\begin{tabular}{ccc}
\hline \multirow{2}{*}{$\mathrm{Características}$} & Profundidade $(\mathrm{cm})$ & \\
\cline { 2 - 3 } $\mathrm{pH}$ & $0-20$ & $20-40$ \\
\hline $\mathrm{H}^{+}\left(\mathrm{cmol}^{\mathrm{c}} \mathrm{dm}^{-3}\right)$ & 5,1 & 4,4 \\
$\mathrm{Al}^{3+}\left(\mathrm{cmol}^{\mathrm{c}} \mathrm{dm}^{-3}\right)$ & 0,1 & 6,8 \\
$\mathrm{Ca}^{2+}\left(\mathrm{cmol}^{\mathrm{c}} \mathrm{dm}^{-3}\right)$ & 3,2 & 1,2 \\
$\mathrm{Mg}^{2+}\left(\mathrm{cmol}^{\mathrm{c}} \mathrm{dm}^{-3}\right)$ & 1,4 & 1,4 \\
$\mathrm{~K}^{+}\left(\mathrm{cmol}^{\mathrm{c}} \mathrm{dm}^{-3}\right)$ & 0,3 & 0,6 \\
$\mathrm{CTC}_{\mathrm{pH}}\left(\mathrm{cmol}^{\mathrm{c}} \mathrm{dm}^{-3}\right)$ & 9,1 & 0,1 \\
$\mathrm{C} . \mathrm{O} .\left(\mathrm{g} \mathrm{dm}^{-3}\right)$ & 15,8 & 10,1 \\
$\mathrm{P}_{\mathrm{resina}}\left(\mathrm{mg} \mathrm{dm}^{-3}\right)$ & 19,7 & 12,2 \\
$\mathrm{Fe}\left(\mathrm{mg} \mathrm{dm}^{-3}\right)$ & 9,0 & 2,0 \\
$\mathrm{Cu}\left(\mathrm{mg} \mathrm{dm}^{-3}\right)$ & 7,2 & 5,0 \\
$\mathrm{Zn}\left(\mathrm{mg} \mathrm{dm}^{-3}\right)$ & 2,0 & 5,0 \\
$\mathrm{~B}\left(\mathrm{mg} \mathrm{dm}^{-3}\right)$ & 0,2 & 0,1 \\
$\mathrm{Mn}\left(\mathrm{mg} \mathrm{dm}^{-3}\right)$ & 25,3 & 0,1 \\
$\mathrm{H}^{+} \%$ & 44,8 & 6,2 \\
$\mathrm{Al}^{3+} \%$ & 0,9 & 67,5 \\
$\mathrm{Ca}^{2+} \%$ & 35,7 & 12,2 \\
$\mathrm{Mg}^{2+} \%$ & 15,6 & 14,3 \\
$\mathrm{~K}^{+} \%$ & 3,0 & 5,5 \\
$\mathrm{~V}^{+} \%$ & 54,3 & 20,3 \\
\hline
\end{tabular}

Tabela 2. Dados pluviométricos durante o ciclo da cultura de trigo no ano de 2004, em Cândido Mota, SP.

\begin{tabular}{ccccc}
\hline Quinzena & Maio & Junho & Julho & Agosto \\
\hline $0-15$ & $48 \mathrm{~mm}$ & $72 \mathrm{~mm}$ & $35 \mathrm{~mm}$ & $0 \mathrm{~mm}$ \\
$16-30$ & $89 \mathrm{~mm}$ & $1 \mathrm{~mm}$ & $114 \mathrm{~mm}$ & $0 \mathrm{~mm}$ \\
\hline
\end{tabular}


Utilizou-se delineamento experimental inteiramente casualizado com quatro repetições. As parcelas possuiam 2 × 6 metros, utilizando como área útil central na parcela 7,5 m² (1,5 x $5 \mathrm{~m})$. Realizaramse três tratamentos: MONITORADO - Adubação de semeadura com $250 \mathrm{~kg} \mathrm{ha}^{-1}$ de 08-20-20, e complementação nutricional via foliar, através da diagnose foliar (DRIS), a cada 30 dias após a emergência, realizadas manualmente com o uso de pulverizador costal a $\mathrm{CO}_{2}$; REGIONAL - adubação de semeadura com $250 \mathrm{~kg} \mathrm{ha}^{-1}$ de 08-20-20, mais 40 $\mathrm{kg} \mathrm{ha}{ }^{-1}$ de nitrogênio, na forma $\left(\mathrm{NH}_{4}\right)_{2} \mathrm{SO}_{4} \mathrm{em}$ cobertura, aplicado a lanço aos 30 dias de emergido e TESTEMUNHA - adubação de semeadura de $250 \mathrm{~kg} \mathrm{ha}^{-1}$ de 08-20-20.

Foram coletas folhas na área útil central da parcela nos três tratamentos, nos períodos correspondentes aos estádios fisiológicos indicados por Malavolta, Vitti e Oliveira(1997) para acompanhar as curvas de absorção dos nutrientes e diagnose foliar (DRIS). A primeira coleta foi realizada aos trinta dias de emergência, a segunda, trinta dias após a primeira, e a terceira, 30 dias após a segunda, (30, 60 e 90 dae). $\mathrm{Na}$ amostragem das folhas, coletaram-se 30 folhas por tratamento (segunda folha expandida de cima para baixo) amostradas ao acaso em todas as repetições, posteriormente acondicionadas em sacos de papel e levadas ao laboratório. Foi realizada análise de macro e micro nutrientes nas folhas segundo Eutroférrico, Empresa Brasileira de Pesqisa Agropecuária (1997).

Na colheita, utilizando-se arcos metálicos de 1,5 x $5 \mathrm{~m}$, para delimitar a área útil da parcela, a colheita se fez com facão e posterior trilhagem em trilhadeira elétrica. As amostras foram pesadas e medida a umidade, corrigindo-se para a umidade de $11 \%$.
Após a colheita, foram retiradas amostras compostas de solo, com o auxílio de um trado, em cada repetição e estratificadas em: 0-5 cm; $5-10 \mathrm{~cm}$; 10-20 cm e 20-40 cm, cujas características químicas foram analisadas segundo Pavan et al. (1992).

O programa estatístico Sisvar foi utilizado para avaliar a comparação de médias pelo teste de Tukey a 5\%, para: número de grãos inteiros por espiga, peso de mil grãos, altura de planta e produtividade. $\mathrm{O}$ comportamento de doenças foi avaliado visualmente.

\section{Resultados e Discussão}

Os resultados do índice DRIS da primeira coleta de folhas (30 dae), para os tratamentos indicaram deficiência de Ca e B (Tabela 3), que pode ser observado pelo valor negativo dos índices para os elementos, pois quanto mais negativo os índices em relação ao Índice de matéria Seca (IMS), mais deficiente a planta se apresenta. A análise do solo da cultura instalada (Tabela 1) revelou o desbalanço da saturação do complexo de troca, com o cálcio ocupando aproximadamente $35,7 \%$ da CTC, sendo que o recomendado para cálcio seria de 50 a $60 \%$ (MARIANO; OLIVEIRA, 2001). A deficiência de boro é justificada pelo baixo teor no solo ou ainda através da inibição não competitiva do nitrogênio (MALAVOLTA; KLIEMANN, 1985). Desta forma, aplicou-se $290 \mathrm{~g} \mathrm{ha}^{-1}$ de cálcio e $60 \mathrm{~g} \mathrm{ha}^{-1}$ boro, na forma de $\mathrm{H}_{3} \mathrm{BO}_{4}$ (IAC, 2002) no tratamento monitorado, via foliar 30 dae. O nitrogênio e o enxofre revelaram-se deficientes nos tratamentos, Regional e Testemunha e apenas a Testemunha apresentou deficiência de magnésio na avaliação de 30 dae. 
Tabela 3. Índice de Matéria Seca (IMS), Índice de Balanço Nutridional (IBN) e Índice DRIS para os nutrientes foliares do trigo nos tratamentos com coletas em dias após emergência (dae)

\begin{tabular}{ccccccccccccccc}
\hline Tratamentos & Coleta & IMS & IBN & $\mathrm{N}$ & $\mathrm{P}$ & $\mathrm{K}$ & $\mathrm{Ca}$ & $\mathrm{Mg}$ & $\mathrm{S}$ & $\mathrm{B}$ & $\mathrm{Cu}$ & $\mathrm{Fe}$ & $\mathrm{Mn}$ & $\mathrm{Zn}$ \\
\hline Monitorado & 30 & $-2,4$ & 20,1 & $-0,3$ & 0,0 & 2,1 & $-3,9$ & $-0,6$ & $-2,4$ & $-3,4$ & 2,3 & 4,9 & 0,1 & 4,2 \\
& 60 & $-2,1$ & 41,6 & $-1,8$ & $-1,0$ & 1,6 & $-1,7$ & $-0,3$ & $-1,2$ & $-2,2$ & 4,9 & $-1,5$ & 2,1 & 3,0 \\
& 90 & $-2,6$ & 35,8 & 1,1 & $-4,2$ & $-1,9$ & 3,3 & 3,0 & 0,5 & $-2,9$ & 0,2 & $-2,6$ & 0,6 & $-0,2$ \\
\hline Regional & 30 & $-2,0$ & 30,3 & $-2,2$ & 0,8 & 2,4 & $-6,0$ & $-1,7$ & $-2,1$ & $-2,7$ & 2,4 & 9,1 & 1,3 & 2,1 \\
& 60 & $-0,6$ & 30,4 & $-1,9$ & $-1,6$ & 3,4 & $-2,0$ & $-0,3$ & $-0,5$ & $-1,8$ & 1,7 & $-2,0$ & 1,3 & 3,1 \\
& 90 & $-0,4$ & 29,7 & $-6,7$ & $-0,9$ & 0,1 & 5,2 & 1,8 & 0,4 & $-2,4$ & $-0,9$ & $-2,7$ & 2,3 & 1,7 \\
\hline Testemunha & 30 & 0,8 & 27,9 & $-1,1$ & 0,3 & 3,0 & $-9,2$ & $-1,7$ & $-2,0$ & $-4,1$ & 2,4 & 10,0 & 1,5 & 4,0 \\
& 60 & 1,4 & 24,6 & $-0,2$ & $-1,0$ & 0,7 & $-4,4$ & $-0,2$ & $-1,2$ & $-1,3$ & 2,7 & $-1,6$ & 2,9 & 2,8 \\
& 90 & 0,7 & 24,7 & $-6,7$ & $-1,7$ & 0,0 & 4,6 & 2,3 & 0,4 & $-1,8$ & $-0,4$ & $-2,9$ & 2,1 & 4,0 \\
\hline
\end{tabular}

Na segunda amostragem aos 60 dae, observouse que o tratamento Monitorado respondeu às doses de cálcio e boro aplicadas aos 30 dae. Embora se tenha notado uma redução significativa no índice para elemento boro, ele continuou apresentando-se deficiente. De acordo com os dados pluviométricos (Tabela 2), a chuva pode ter contribuído para a lixiviação e reduzido a absorção do nutriente. Assim para suprir a necessidade do elemento foi realizada mais uma aplicação de $60 \mathrm{~g} \mathrm{ha}^{-1}$ de boro. Apesar do nitrogênio não apresentar deficiência, seu índice está bem próximo do IMS, e como a planta demanda grande teor do nutriente, foi realizada uma complementação, na qual aplicou-se $40 \mathrm{~kg} \mathrm{ha}^{-1}$ de nitrogênio, na forma $\left(\mathrm{NH}_{4}\right)_{2} \mathrm{SO}_{4}$. De acordo com Ruiz (2001) a falta de nitrogênio pode ser superada fornecendo o nitrogênio em doses parceladas, onde se pode observar aumentos na absorção pós-floração.

No tratamento Regional, 60 dae, os elementos nitrogênio, fósforo, cálcio, boro e ferro apresentaramse deficientes. A aplicação de nitrogênio na forma de sulfato aos 30 dae induziu maior absorção dos elementos nitrogênio e enxofre, como conseqüência um maior requerimento do fósforo seria necessário. Como este não esteve prontamente disponível, ocorreu uma deficiência do mesmo, o que também limitou o nitrogênio a este equilíbrio, ocasionando também maior deficiência em outros elementos, inclusive o ferro, o qual não havia apresentado deficiência aos 30 dae. Folhas com deficiência de ferro são caracterizadas pelo baixo teor de amido e açúcares, pelo fato de cessar o desenvolvimento de cloroplastos (MARSCHNER, 1995). No entanto, observou-se que o enxofre deixou de ser deficiente, o que indica a importância da fonte na correção de deficiências das plantas. O tratamento Testemunha apresentou deficiência em ordem decrescente para cálcio, ferro, boro, enxofre, fósforo, magnésio e nitrogênio.

Os índices DRIS na terceira amostragem foliar, 90 dae, para os tratamentos; Regional e Testemunha, apresentaram deficiência para os elementos, nitrogênio, fósforo, boro, cobre e ferro. Nestes tratamentos o elemento mais limitante foi o nitrogênio, com o maior índice negativo. No tratamento Monitorado devido às intervenções nutricionais observou-se que o nitrogênio deixou de ser um elemento limitante, este resultado corrobora com a necessidade da aplicação do nutriente (HUSSAIN, 1996). No entanto, é necessário observar não só a necessidade nutricional de apenas um elemento, e sim a necessidade global da planta, neste caso a análise do DRIS é um ótimo indicativo, desde que realizadas as análises nos períodos corretos. Desta forma observou-se que aos 90 dae o fósforo e novamente o boro apresentaram deficiência. Uma resposta à deficiência de boro é a inibição do crescimento da raiz, ficando esta com uma aparência 
curta e grossa, e também ficando prejudicado o transporte de cálcio (FURLANI; CARVALHO; FREITAS, 2003). A intervenção foi realizada com a aplicação de $250 \mathrm{~g} \mathrm{ha}^{-1}$ de fósforo e $60 \mathrm{~g} \mathrm{ha}^{-1}$ de boro.

Sem a análise do DRIS as observações de deficiências nutricionais na cultura do trigo não poderiam ter sido detectadas, a Tabela 4 trás os teores dos nutrientes foliares de forma isolada, em que não é possível verificar a necessidade nutricional da planta. De acordo com Malavolta; Vitti e Oliveira (1997) a interpretação feita comparando-se os resultados analíticos com parâmetros previamente tabelados apresenta desvantagem, pois os nutrientes são avaliados individualmente, não levando em consideração as interações existentes.

Tabela 4. Teores dos nutrientes foliares do trigo nos tratamentos, com coletas em dias após emergência (dae).

\begin{tabular}{|c|c|c|c|c|c|c|c|c|c|c|c|c|}
\hline \multirow[b]{2}{*}{ Tratamentos } & \multirow[b]{2}{*}{ Coleta } & $\mathrm{N}$ & $\mathrm{P}$ & $\bar{K}$ & $\mathrm{Ca}$ & $\mathrm{Mg}$ & $S$ & B & $\mathrm{Cu}$ & $\mathrm{Fe}$ & $\mathrm{Mn}$ & $\mathrm{Zn}$ \\
\hline & & \multicolumn{6}{|c|}{$\mathrm{g} \mathrm{kg}^{-1}$} & \multicolumn{5}{|c|}{$\mathrm{mg} \mathrm{kg}^{-1}$} \\
\hline \multirow[t]{3}{*}{ Monitorado } & 30 & 59,9 & 5,9 & 43,4 & 2,7 & 1,8 & 4,5 & 9,6 & 15,2 & 928,7 & 111,8 & 52,1 \\
\hline & 60 & 52,3 & 6,3 & 44,0 & 1,5 & 1,4 & 3,6 & 8,2 & 12,6 & 1295,0 & 150,7 & 48,1 \\
\hline & 90 & 48,0 & 7,3 & 43,0 & 2,2 & 1,5 & 3,7 & 12,3 & 15,1 & 1441,0 & 155,8 & 42,7 \\
\hline \multirow[t]{3}{*}{ Regional } & 30 & 63,1 & 3,0 & 17,0 & 6,6 & 3,5 & 4,1 & 12,2 & 9,0 & 96,3 & 98,6 & 27,7 \\
\hline & 60 & 30,4 & 6,0 & 30,6 & 3,3 & 1,9 & 5,9 & 2,0 & 3,4 & 84,0 & 67,0 & 79,5 \\
\hline & 90 & 29,7 & 4,0 & 25,3 & 2,8 & 1,7 & 5,5 & 1,7 & 3,0 & 80,0 & 55,0 & 114,5 \\
\hline \multirow[t]{3}{*}{ Testemunha } & 30 & 45,6 & 2,7 & 19,1 & 1,4 & $\overline{1,0}$ & 2,0 & 6,7 & 10,6 & 90,4 & 91,3 & 24,7 \\
\hline & 60 & 39,4 & 2,0 & 18,0 & 1,3 & 1,0 & 2,0 & 7,5 & 9,1 & 106,8 & 108,8 & 24,6 \\
\hline & 90 & 49,1 & 2,8 & 27,5 & 2,2 & 1,3 & 2,4 & 7,5 & 8,9 & 107,5 & 91,9 & 29,9 \\
\hline
\end{tabular}

Nos três tratamentos observou-se visualmente a presença de manchas foliares na fase de perfilhamento, mas não houve necessidade de entrar com controle químico, pois aplicou-se preventivamente fungicida quando a lavoura estava iniciando o período de florescimento.

O tratamento Monitorado da cultura proporcionou diferença na produtividade, no número de grãos inteiros/ espiga e no peso de 1.000 grãos (Tabela 5). A produtividade foi superior em $1.349 \mathrm{~kg} \mathrm{ha}^{-1}$ no tratamento Monitorado em relação à Testemunha e $536 \mathrm{~kg} \mathrm{ha}^{-1}$ a mais que o tratamento Regional. O fornecimento apenas de nitrogênio, como realizado no tratamento Regional, não surte efeito satisfatório para a cultura. Assim, o monitoramento acompanhado pelo método DRIS pode proporcionar o suporte correto para a cultura, com ganhos expressivos na produtividade. $\mathrm{O}$ tratamento Monitorado proporcionou aumento em relação à adubação
Regional de 21,80 \% na produção. Com relação às características agronômicas o tratamento Monitorado, apresentou um acréscimo de 21 grãos inteiros e $2 \mathrm{~g}$ a mais no peso de 1.000 grãos em relação à testemunha e 6,4 grãos inteiros a mais do que o tratamento Regional. Segundo Bredemeier, Mundstock e Büttenbender (2001), a quantidade de $\mathrm{N}$ por semente aumentou com a massa da semente, só que em proporção maior do que a massa seca, pois também o teor de $\mathrm{N}$ da semente incrementou à medida que o tamanho da semente aumentou.

A análise estratificada do solo no final do experimento (Tabela 6) mostra a deficiência de cálcio, assim como na análise realizada no início do experimento. O potássio encontra-se um pouco acima do teor indicado para os tratamentos Monitorado e Testemunha nos cinco primeiros centímetros, que deveria estar entre 3 a $5 \%$, conforme recomendado por Lopes; Guilherme (1992). As concentrações de 
magnésio para os primeiros vinte centímetros do tratamento Monitorado e nos cinco primeiros centímetros do tratamento Testemunha apresentaram-se um pouco acima do recomendado, que seria entre $10-15 \%$ do complexo de troca (MARIANO; OLIVEIRA, 2001). A percentagem de alumínio apresentou-se alta para todos os tratamentos na profundidade de $20-40 \mathrm{~cm}$.

Tabela 5. Médias de peso 1000 grãos (g), número de grãos inteiros/espiga e produtividade para os tratamentos.

\begin{tabular}{cccc}
\hline Tratamentos & Peso 1000 grãos $(\mathrm{g})$ & $\mathrm{N}^{\mathrm{o}}$ grãos inteiros/espiga & ${\text { Produtividade }\left(\mathrm{kg} \mathrm{ha}{ }^{-1}\right)}$ \\
\hline Monitorado & $41,30 \mathrm{a}^{*}$ & $48,5 \mathrm{a}$ & $2.978 \mathrm{a}$ \\
Regional & $40,30 \mathrm{ab}$ & $42,1 \mathrm{~b}$ & $2.442 \mathrm{~b}$ \\
Testemunha & $39,34 \mathrm{~b}$ & $27,5 \mathrm{c}$ & $1.629 \mathrm{c}$ \\
\hline
\end{tabular}

*Médias seguidas de mesma letra, não diferem entre si pelo teste de Tukey $(\mathrm{p}>0.05)$

Tabela 6. Avaliação da saturação do complexo de troca do perfil do solo para os tratamentos após a colheita do trigo.

\begin{tabular}{cccccccc}
\hline Tratamentos & Profundidade & \multicolumn{7}{c}{ Saturação do Complexo de Troca \% } \\
\hline & $\mathrm{cm}$ & $\mathrm{Ca}^{2+}$ & $\mathrm{Mg}^{2+}$ & $\mathrm{K}^{+}$ & $\mathrm{Al}^{3+}$ & $\mathrm{H}^{+}$ & $\mathrm{V} \%$ \\
\hline \multirow{3}{*}{ Monitorado } & $0-5$ & 35,9 & 18,5 & 5,4 & 0,0 & 40,2 & 59,8 \\
& $5-10$ & 38,5 & 15,9 & 2,5 & 1,1 & 42,0 & 56,9 \\
& $10-20$ & 37,3 & 15,4 & 1,0 & 0,0 & 46,3 & 53,7 \\
& $20-40$ & 16,9 & 6,1 & 0,7 & 12,1 & 64,2 & 23,7 \\
\hline \multirow{3}{*}{ Regional } & $0-5$ & 30,0 & 14,0 & 4,0 & 2,0 & 50,0 & 48,0 \\
& $5-10$ & 34,4 & 15,0 & 3,3 & 1,1 & 46,2 & 52,7 \\
& $10-20$ & 31,0 & 13,6 & 2,1 & 1,2 & 52,1 & 46,7 \\
& $20-40$ & 10,1 & 4,0 & 0,5 & 15,9 & 69,5 & 14,6 \\
\hline \multirow{3}{*}{ Testemunha } & $0-5$ & 33,8 & 16,3 & 5,2 & 1,1 & 43,6 & 55,3 \\
& $5-10$ & 35,7 & 14,3 & 2,5 & 1,2 & 46,3 & 52,5 \\
& $10-20$ & 44,8 & 17,2 & 1,3 & 0,0 & 36,7 & 63,3 \\
& $20-40$ & 15,9 & 6,3 & 0,5 & 8,5 & 68,8 & 22,7 \\
\hline
\end{tabular}

\section{Conclusão}

A interferência nutricional, através do DRIS, na cultura do trigo proporcionou melhorias nutricionais na cultura, bem como aumento de produção.

O uso do método DRIS no monitoramento se mostrou eficiente, como forma de melhoria no fornecimento de nutriente para a cultura do trigo.

\section{Referências}

BEAUFILS, E.R. Diagnosis and recommendation integrated system (dris). Pietermaritzburg: University of Natal, 1973.

BREDEMEIER, C.; MUNDSTOCK, C. M.; BÜTTENBENDER, D. Efeito do tamanho das sementes de trigo no desenvolvimento inicial das plantas e no rendimento de grãos. Pesquisa Agropecuária Brasileira, Brasilia, v.36, n.8, p.1061-1068, 2001.

CAMPBELL, C. A.; DE JONG, R. Root-to-straw influence of moisture and rate of $\mathrm{N}$ fertilizer. Canadian Journal of Plant Science, Ottawa, v. 81, p. 39-43, 2000. 
EMPRESA BRASILEIRA DE PESQUISA AGROPECUÁRIA (EMBRAPA). Sistema brasileiro de classificação de solos. Brasília: Embrapa-CNPS, 1999.

FREITAS, J. G.; CAMARGO, C. E. O.; FERREIRA FILHO, A. W. P.; CASTRO, J. L. Eficiência e resposta de genótipos de trigo ao nitrogênio. Revista Brasileira de Ciências do Solo, Campinas, v.19, p. 229-234, 1995.

FREITAS, J. G.; CANTARELLA, H.; CAMARGO, C. E. O.; FERRIRA FILHO, A. W. P. ; PETTINELLI, A. J.; RAMOS, V. J.; FELICIO, J. C. Resposta ao calcário e ao fósforo de genótipos de trigo com diferentes tolerâncias ao alumínio. Pesquisa Agropecuária Brassileira, Brasília, v.35, n.3, p.557-566, 2000.

FURLANI, A. M. C.; CARVALHO, C. P.; FREITAS, J. G.; VERDIAL, M. F. Wheat cultivar tolerance to boron deficiency and toxicity in nutrient solution. Scientia Agricola, Piracicaba, v.60, n.2, p.359-370, 2003.

HUSSAIN, G; ALJALOUD, A. A; KARIMULLA, S. Effect of treated effluent irrigation and nitrogen on yield and nitrogen use efficiency of wheat. Agricultural Water Management, Amsterdam, v.30, n.2, p.175-184, 1996.

COMISSÕES CENTRO-SUL BRASILEIRA DE PESQUISA DE TRIGO E TRITICALE. Informações Técnicas das para a safra de 2004. In: REUNIÃO DA COMISSÃO CENTROSUL BRASILEIRA DE PESQUISA DE TRIGO, 19., 2004, Londrina. Anais... Londrina, 2004. (Documento, n.1).

INSTITUTO AGRONÔMICO DE CAMPINAS. Recomendações da Comissão Técnica de Trigo para 2002. Campinas, 2002.
LOPES, A. S.; GUILHERME, L. R. G. Solos sob cerrado: Manejo da fertilidade para a produção agropecuária. São Paulo: ANDA, 1994. (Boletim Técnico, n.5)

MALAVOLTA, E.; KLIEMANN, H. J. Desordens nutricionais no nerrado. Piracicaba: Potafós, 1985.

MALAVOLTA, E.; VITTI, G. C; OLIVEIRA, S. A. Avaliação do estado nutricional das plantas: princípios e aplicações. 2.ed. Piracicaba: Potafós, 1997.

MARIANO, J. C.; OLIVEIRA, E. F. Fertilidade do solo e nutrição de plantas. Campo Mourão: COAMO, 2001.

MARSCHNER, H. Mineral Nutrition of Higher Plants. 2.ed. New York: Academic Press, 1995.

PAULETTI, V. Plantio direto: atualização tecnológica. São Paulo: Fundação Cargil, 1998.

PAVAN, M. A.; BLOCH, M. D. M.; ZEMPULSK, H. C.; MIYAZAWA, M.; ZOCOLER, D. C. Manual de análises químicas de solo e controle da qualidade. Londrina: IAPAR, 1992. (Circular Técnica, n.76).

RUIZ, R. A. Requerimientos de nutrientes y fertilización del cultivo. área de comunicaciones de asociación argentina de consorcios regionales de experimentación agrícola, Siembra Directa. Buenos Aires: AACREA, 2001. p.24-33. (Cuaderno de Actualización Técnica, 63).

SILVA, E.B.; NOGUEIRA, F. D.; GUIMARÃES, P. T. G. Uso do DRIS na avaliação do estado nutricional do cafeeiro em resposta à adubação potássica. Revista Brasileira de Ciências do Solo, Campinas, v.27, p.247255, 2003. 